\title{
Clinical Study of Prevalence of Antibiotic Resistance of Escherichia coli in Urinary Tract Infection in Children: A 9-year Retrospective, Single Center Experience
}

\section{Eun Young Seo, M.D. Seung Man Cho, M.D., Ph.D. Dong Seok Lee, M.D., Ph.D. Sung Min Choi, M.D., Ph.D. Doo Kwun Kim, M.D., Ph.D.}

Department of Pediatrics, Dongguk University Gyeongju Hospital, Dongguk University, Gyeongju, Republic of Korea

\section{Corresponding author:}

Doo Kwun Kim, M.D., Ph.D. University Gyeongju Hospital, Dongguk University, 87, Dongdae-ro, Gyeongju-si, Gyeongsangbuk-do, Republic of Korea

Tel: $+82-54-770-8254$

Fax: +82-54-770-8500

E-mail: pedepi@hotmail.com

Received: 30 August 2017

Revised: 21 September 2017

Accepted: 28 September 2017
Department of Pediatrics, Dongguk

Purpose: The aim of this study was to determine the prevalence of antibiotic susceptibility and resistance of Escherichia coli in urinary tract infections (UTIs) in children.

Methods: We retrospectively reviewed the clinical records of 212 inpatients aged 18 years or younger with UTIs treated at the Pediatric Department of Dongguk University Gyeongju Hospital between January 2008 and December 2016. For comparison, patients were divided into three groups according to age as follows: group 1, $\leq 1$ month; group 2, $>1$ month to $\leq 12$ months; and group 3, $\geq 13$ months. The antibiotic resistance rates from January 2008 to December 2012 (study period 1) and from January 2013 to December 2016 (study period 2) were analyzed statistically by group.

Results: As the patient age increased, the antibiotic resistance rate to ampicillin $(P=0.013)$, levofloxacin $(P=0.050)$, piperacillin/tazobactam (TZP) $(P<0.001)$, and trimethoprim/sulfamethoxazole $(P=0.002)$ increased. The frequency of extended spectrum beta-lactamase producing $E$. coli showed a significant difference from 5 cases (4.6\%) in study period 1 and 16 cases (15.8\%) in study period $2(P=0.007)$. The antibiotic resistance rate of $E$. coli was compared between the two time periods and we found that the antibiotic resistance rate to cefotaxime was significantly increased from $5.4 \%$ to $16.8 \%(P=0.008)$ and that to TZP was significantly decreased from $40.5 \%$ to $7.9 \%(P<0.001)$.

Conclusion: Over the past 9 years, the resistance rate to cefotaxime has increased but the resistance rate to TZP has decreased. Thus, it is important to continue to investigate the antibiotic resistance rates of bacteria in the community.

Key words: Antibiotic resistance, Urinary tract infection, Escherichia coli

\section{Introduction}

Although urinary tract infections (UTIs) are one of the most common under the terms of the Creative Commons Attribution Non-Commercial License (http:// creativecommons.org/licenses/by-nc/4.0/) which permits unrestricted non-commercial use, distribution, and reproduction in any medium, provided the original work is properly cited. childhood bacterial infectious diseases, in many cases its diagnosis is delayed due to non-specific symptoms. Failure of timely treatment can result in sequela related to kidney injury, including renal scarring, hypertension, and chronic kidney disease ${ }^{1)}$. Thus, it is important to choose appropriate antibiotics, depending on pathogen susceptibility testing results. However, it is often difficult to isolate pathogens in children due to false positive and false nega- 
tive results, depending on the urine collection method. Additionally, it takes a substantial amount of time to obtain the results of antibiotic susceptibility testing, so that selection of initial antibiotics is dependent on empirical antibiotics.

Recently, extended antibiotic use brought about changes in causative strains of UTIs and rates of antibiotic susceptibility ${ }^{2,3)}$. In particular, an increasing number of strains produce extended spectrum $\beta$-lactamase $(\mathrm{ESBL})^{4,5)}$. The present study was aimed to propose a direction for future UTI treatment by identifying UTI pathogens in children, investigating the changing trends in antibiotic resistance, and incidence of the most common pathogen: ESBL-producing E. coli.

\section{Materials and methods}

\section{Subjects}

We identified pediatric patients, 18 years or younger, who were admitted to the Pediatrics Clinic of Dongguk University Gyeongju Hospital from January 2008 to December 2016, and then diagnosed with UTI. Of these, 212 patients who exhibited $E$. coli were selected as subjects.

\section{Methods}

Inclusion criteria were pediatric patients who had fever $38.0^{\circ} \mathrm{C}$ or higher and pyuria, with at least five white blood cells in urine/high-power field (HPF), leading to clinical suspicion of UTI, or patients whose urine culture test produced $E$. coli in at least $10^{5}$ colony-forming units (CFU)/ $\mathrm{mL}$. Based on these findings, we determined these patients had UTIs caused by E. coli. Patients with two or more strains on culture were excluded.

For urine collection, the urethrae externum was cleaned with boric acid and distilled water. Thereafter, a clean catch midstream urine sample was collected from children who were potty-trained, while the sterile urine collection bag method or catheterization method was used to collect urine from infants who were not potty-trained. For urine culture, $0.001 \mathrm{~mL}$ of collected urine was inoculated on a $5 \%$ sheep blood agar plate and MacConkey agar plate, followed by incubation at $36^{\circ} \mathrm{C}$ within a $5 \% \mathrm{CO}_{2}$ incubator for $48 \mathrm{hrs}$. We then analyzed all formed colonies.

We examined the gender distribution and age in pediatric patients with $E$. coli UTIs, and then retrospectively analyzed antibiotic susceptibility of the $E$. coli, depending on ESBL production. For comparative analysis by age, we divided patients into three groups aged: younger than 1 month; 1-12 months, and 13 months or older. To balance the sample number between periods, we compared 111 cases from January 2008 to December 2012 (study period 1) and 101 cases from January 2013 to December to 2016 (study period 2).

Antibiotic susceptibility test was performed using the VITEK $^{\circledR}-2$ Antimicrobial Susceptibility Test (AST) card (Bio-Mérieux, Marcy l'Etoile, France), an automatic device for bacterial identification and measurement of antibiotic susceptibility. As a result, antibiotic susceptibility was divided into resistance, intermediate resistance and susceptibility, with intermediate resistance regarded as resistance for the purposes of statistical analysis.

For the ESBL test, we used the VITEK ${ }^{\circledR}-2$ ESBL test using VITEK $^{\circledR}-2$ Antimicrobial Susceptibility Test card. The panel consisted of six containers, where three containers contained $0.5 \mathrm{mg} / \mathrm{L}$ ceftazidime, $0.5 \mathrm{mg} / \mathrm{L}$ cefotaxime, and $1.0 \mathrm{mg} / \mathrm{L}$ cefepime, respectively, and the remaining three containers held either $4 \mathrm{mg} / \mathrm{L}, 4 \mathrm{mg} / \mathrm{L}$ or $10 \mathrm{mg} / \mathrm{L}$ of clavulanic acid in addition to $0.5 \mathrm{mg} / \mathrm{L}$ ceftazidime, $0.5 \mathrm{mg} / \mathrm{L}$ cefotaxime and $1.0 \mathrm{mg} / \mathrm{L}$ cefepime, respectively. Unlike the containers that only contained cephalosporins, if bacterial cultures were inhibited only in the containers with both cephalosporins and clavulanic acid, the strain was considered as an ESBL-producing strain. In cases where there was at least one positive result, out of the three containers, the strain was confirmed as an ESBL-producing strain.

\section{Statistical analysis}

We used the Korean version of SPSS 18.0 for windows program, and applied analytical methods for each item including the Pearson chi-square, chi-square test for trend, and Fisher's exact test. The significance level was set as $P$ value $<0.05$.

\section{Results}

\section{Characteristics of subjects}

There were 212 pediatric patients, consisting of 146 boys 
(68.9\%) and 66 girls (31.1\%), showing a 2.2:1 gender ratio. Gender ratios by age group were 2.2:1 for patients younger than 1 month of age, 3.3:1 for patients who were 1-12 months of age, and 1:1.3 for the group who were 13 months old or older. For gender-based differences in antibiotic resistance, boys were significantly more resistant to ampicillin $(P=$ $0.030)$, gentamicin $(P=0.016)$, and TMP/SMX $(P<0.001)$ than girls (Table 1).

We further divided the patients into three groups by age as follows: younger than 1 month ( $\mathrm{n}=29), 1-12$ months $(\mathrm{n}=$ $138)$, and 13 months or older $(n=45)$. Antibiotics which resistance rate increased with age included: amoxicillin/ clavulanate $(P<0.001)$, ampicillin $(P=0.013)$, levofloxacin $(P=0.050)$, TZP $(P<0.001)$, and TMP/SMX $(P=0.002)$ (Table 2).

Table 1. Demographic Findings of Study Population

\begin{tabular}{|c|c|c|c|}
\hline & $\begin{array}{c}\text { Male } \\
(N=146)\end{array}$ & $\begin{array}{l}\text { Female } \\
(\mathrm{N}=66)\end{array}$ & $\begin{array}{c}P_{-} \\
\text {value }\end{array}$ \\
\hline Number & 146 & 66 & \\
\hline \multicolumn{4}{|l|}{ Age } \\
\hline Mean \pm S.D. & $8.13 \pm 17.06$ & $23.10 \pm 33.40$ & \\
\hline Underlying urogenital tract anomaly & 6 & 3 & \\
\hline Catheter related infection & 0 & 0 & \\
\hline \multicolumn{4}{|l|}{ Episode } \\
\hline First & 126 & 62 & \\
\hline Recurrence & 20 & 4 & \\
\hline \multicolumn{4}{|l|}{ Kidney sono } \\
\hline Normal & 92 & 36 & \\
\hline Abnormal & 37 & 12 & \\
\hline Overall ESBL(+) E.coli prevalence & 12 & 9 & \\
\hline \multicolumn{4}{|l|}{ Antibiotics resistance rate } \\
\hline Amikacin & $2(1.4 \%)$ & $1(1.5 \%)$ & 1.000 \\
\hline Amoxicillin/Clavulanate & $36(24.7 \%)$ & $17(25.8 \%)$ & 0.864 \\
\hline Ampicillin & $81(55.5 \%)$ & $47(71.2 \%)$ & 0.030 \\
\hline Aztreonam & $13(8.9 \%)$ & $9(13.6 \%)$ & 0.295 \\
\hline Cefepime & $13(8.9 \%)$ & $9(13.6 \%)$ & 0.295 \\
\hline Cefotaxime & $14(9.6 \%)$ & $9(13.6 \%)$ & 0.380 \\
\hline Cefoxitin & $4(2.7 \%)$ & $3(4.5 \%)$ & 0.680 \\
\hline Ceftazidime & $14(9.6 \%)$ & $9(13.6 \%)$ & 0.380 \\
\hline Gentamicin & $12(8.2 \%)$ & $13(19.7 \%)$ & 0.016 \\
\hline Imipenem & $0 \%$ & $0 \%$ & \\
\hline Levofloxacin & $15(14.2 \%)$ & $8(15.7 \%)$ & 0.199 \\
\hline Meropenem & $0 \%$ & $0 \%$ & \\
\hline Piperacillin/tazobactam & $34(23.3 \%)$ & $19(28.8 \%)$ & 0.392 \\
\hline Trimethoprim/Sulfamethoxazole & $20(13.7 \%)$ & $25(37.9 \%)$ & 0.000 \\
\hline
\end{tabular}

\section{Changes in antibiotic resistance rate of $E$. coli in 2008-2012 and in 2013-2016}

We investigated changes in rates of antibiotic resistance for E. coli isolated 111 cases in 2008-2012 (study period 1) and 101 cases in 2013-2016 (study period 2). The male to female ratio of the two periods was 2:1 in study period 1 and 2.48:1 in study period 2. And the distribution in number of age groups was 1Mo:1-12Mo: $\geq 13$ Mo 1:6.2:2.1 in study period 1, and 1:3.8:1.2 in study period 2 respectively. We found that resistance to cefotaxime increased from 5.4\% to $16.8 \%$ ( $P=0.008)$, whereas resistance to TZP decreased from $40.5 \%$ to $7.9 \%(P<0.001)$. Although they were not statistically significant, resistance to aztreonam and cefepime increased from $5.4 \%$ to $15.8 \%(P=0.13)$, to levofloxacin increased from $8.9 \%$ to $17.8 \%(P=0.13)$, and to ceftazidime increased from $6.3 \%$ to $15.8 \%(P=0.26)$ (Table 3$)$.

\section{Incidence of ESBL producing $E$. coli}

As age increased, frequencies of ESBL-producing E. coli were $10.3 \%$ for patients younger than 1 month, $8.8 \%$ for patients aged 1-12 months, and $13.6 \%$ for patients 13 months old or older (in which there was no statistically significant

Table 2. Antibiotic Resistance Rate and Emergence Frequency of ESBL-producing E. coli according to Age Group

\begin{tabular}{|c|c|c|c|c|}
\hline & $\begin{array}{c}\leq 1 \\
\text { month } \\
(n=29)\end{array}$ & $\begin{array}{l}>1 \text { month to } \\
\leq 12 \text { months } \\
\quad(n=138)\end{array}$ & $\begin{array}{c}\geq 13 \\
\text { months } \\
(n=45)\end{array}$ & $\begin{array}{c}P_{-} \\
\text {value }\end{array}$ \\
\hline \multicolumn{5}{|l|}{ Antibiotic resistance rate } \\
\hline Amikacin & $0(0 \%)$ & $1((0.7 \%)$ & $2(4.4 \%)$ & 0.079 \\
\hline Amoxicillin/Clavulanate & $3(10.3 \%)$ & $28(20.3 \%)$ & $22(48.9 \%)$ & 0.000 \\
\hline Ampicillin & $13(44.8 \%)$ & $82(59.4 \%)$ & $33(73.3 \%)$ & 0.013 \\
\hline Aztreonam & $3(10.3 \%)$ & $13(9.4 \%)$ & $6(13.3 \%)$ & 0.608 \\
\hline Cefepime & $3(10.3 \%)$ & $13(9.4 \%)$ & $6(13.3 \%)$ & 0.608 \\
\hline Cefotaxime & $3(10.3 \%)$ & $13(9.4 \%)$ & $7(15.6 \%)$ & 0.395 \\
\hline Cefoxitin & $0(0 \%)$ & $4(2.9 \%)$ & $3(6.7 \%)$ & 0.106 \\
\hline Ceftazidime & $3(10.3 \%)$ & $14(10.1 \%)$ & $6(13.3 \%)$ & 0.635 \\
\hline Gentamicin & $5(17.2 \%)$ & $12(8.7 \%)$ & $8(17.8 \%)$ & 0.687 \\
\hline Imipenem & $0 \%$ & $0 \%$ & $0 \%$ & \\
\hline Levofloxacin & $1(4.8 \%)$ & $14(13.7 \%)$ & $8(23.5 \%)$ & 0.050 \\
\hline Meropenem & $0 \%$ & $0 \%$ & $0 \%$ & \\
\hline Piperacillin/tazobactam & $2(6.9 \%)$ & $32(23.2 \%)$ & $19(42.2 \%)$ & 0.000 \\
\hline $\begin{array}{l}\text { Trimethoprim/ } \\
\text { Sulfamethoxazole }\end{array}$ & $5(17.2 \%)$ & $21(15.2 \%)$ & $19(42.2 \%)$ & 0.002 \\
\hline \multicolumn{5}{|l|}{ Frequency of $\operatorname{ESBL}(+)$ E. coli } \\
\hline ESBL $(+)$ E. coli & $3(10.3 \%)$ & $12(8.8 \%)$ & $6(13.6 \%)$ & 0.556 \\
\hline
\end{tabular}

Abbreviation: ESBL, extended spectrum beta-lactamase. 
difference) (Table 2).

Incidences of ESBL-producing $E$. coli included 5 cases (4.6\%) in study period 1 and 16 cases (15.9\%) in study period 2 , showing a significant increase $(P=0.007)$ (Table 3$)$.

\section{Antibiotic susceptibility depending on ESBL production}

Within the study period, there were 189 cases of ESBL non-producing $E$. coli. There was no measured resistance to aztreonam, cefepime, imipenem, and meropenem, while resistance to TMP/SMX (18.0\%), amoxicillin/clavulanate (21.2\%) and ampicillin (55.6\%) was high. There were 21 cases of ESBL-producing E. coli that were $100 \%$ susceptible to imipenem and meropenem, and $100 \%$ resistant to am-

Table 3. Antibiotic Resistance Rate Changes and Emergence Frequency of ESBL-producing E. coli Compared with 2008-2012 and 2013-2016

\begin{tabular}{|c|c|c|c|}
\hline & $\begin{array}{c}\text { Study period } 1 \\
2008-2012 \\
(N=111)\end{array}$ & $\begin{array}{c}\text { Study period } 2 \\
2013-2016 \\
(N=101)\end{array}$ & $\begin{array}{c}P- \\
\text { value }\end{array}$ \\
\hline \multicolumn{4}{|l|}{ Sex } \\
\hline Male & 74 & 72 & \\
\hline Female & 37 & 29 & \\
\hline \multicolumn{4}{|l|}{ Age } \\
\hline$\leq 1 \mathrm{month}$ & 12 & 17 & \\
\hline$\geq 1$ month to $\leq 12$ months & 74 & 64 & \\
\hline$\geq 13$ months & 25 & 20 & \\
\hline \multicolumn{4}{|l|}{ Antibiotic resistance rate } \\
\hline Amikacin & $2(1.8 \%)$ & $1(1.0 \%)$ & 1.000 \\
\hline Amoxicillin/Clavulanate & $26(23.4 \%)$ & 27 (26.7\%) & 0.578 \\
\hline Ampicillin & $71(64.0 \%)$ & $57(56.4 \%)$ & 0.263 \\
\hline Aztreonam & $6(5.4 \%)$ & $16(15.8 \%)$ & 0.130 \\
\hline Cefepime & $6(5.4 \%)$ & $16(15.8 \%)$ & 0.130 \\
\hline Cefotaxime & $6(5.4 \%)$ & $17(16.8 \%)$ & 0.008 \\
\hline Cefoxitin & $2(1.8 \%)$ & $5(5.0 \%)$ & 0.262 \\
\hline Ceftazidime & $7(6.3 \%)$ & $16(15.8 \%)$ & 0.260 \\
\hline Gentamicin & $11(9.9 \%)$ & $14(13.9 \%)$ & 0.373 \\
\hline Imipenem & $0 \%$ & $0 \%$ & \\
\hline Levofloxacin & $5(8.9 \%)$ & $18(17.8 \%)$ & 0.131 \\
\hline Meropenem & $0 \%$ & $0 \%$ & \\
\hline Piperacillin/tazobactam & 45 (40.5\%) & $8(7.9 \%)$ & 0.000 \\
\hline Trimethoprim/Sulfamethoxazole & $23(20.7 \%)$ & $22(21.8 \%)$ & 0.850 \\
\hline \multicolumn{4}{|l|}{ Frequency of ESBL(+) E.coli } \\
\hline ESBL (-) E.coli & 104 (95.4\%) & 85 (84.2\%) & \\
\hline ESBL $(+)$ E.coli & $5(4.6 \%)$ & $16(15.8 \%)$ & 0.007 \\
\hline
\end{tabular}

Abbreviation: ESBL, extended spectrum beta-lactamase. picillin, aztreonam, cefepime, cefotaxime and ceftazidime (Table 4).

\section{Difference in antibiotic susceptibility between} patients with first UTI and those with recurrent UTIs Pediatric patients were divided according to UTI onset, including 188 cases of first UTI and 24 cases of recurrent UTIs. Comparing antibiotic resistance rates, we found that the recurrent UTI group showed significantly higher resistance against amikacin $(P=0.034)$, amoxicillin/clavulanate

Table 4. Comparison of Antibiotic Resistance Rates between $\mathrm{ESBL}(-)$ E. coli and $\mathrm{ESBL}(+)$ E. coli

\begin{tabular}{lccc}
\hline & $\begin{array}{c}\text { ESBL }(-) \\
\text { E.coli }(\mathrm{N}=189)\end{array}$ & $\begin{array}{c}\text { ESBL }(+) \\
\text { E.coli }(\mathrm{N}=21)\end{array}$ & $\begin{array}{c}P \text { - } \\
\text { value }\end{array}$ \\
\hline Amikacin & $2(1.1 \%)$ & $1(4.8 \%)$ & 0.272 \\
Amoxicillin/Clavulanate & $40(21.2 \%)$ & $12(57.1 \%)$ & 0.000 \\
Ampicillin & $105(55.6 \%)$ & $21(100 \%)$ & 0.000 \\
Aztreonam & $0(0 \%)$ & $21(100 \%)$ & 0.000 \\
Cefepime & $0(0 \%)$ & $21(100 \%)$ & 0.000 \\
Cefotaxime & $1(0.5 \%)$ & $21(100 \%)$ & 0.000 \\
Cefoxitin & $5(2.6 \%)$ & $2(9.5 \%)$ & 0.147 \\
Ceftazidime & $1(0.5 \%)$ & $21(100 \%)$ & 0.000 \\
Gentamicin & $17(9.0 \%)$ & $7(33.3 \%)$ & 0.004 \\
Imipenem & $0 \%$ & $0 \%$ & \\
Levofloxacin & $16(11.7 \%)$ & $7(35.0 \%)$ & 0.013 \\
Meropenem & $0 \%$ & $0 \%$ & \\
Piperacillin/tazobactam & $43(22 . .8 \%)$ & $8(38.1 \%)$ & 0.120 \\
Trimethoprim/Sulfamethoxazole & $34(18.0 \%)$ & $10(47.6 \%)$ & 0.004 \\
\hline
\end{tabular}

Abbreviation: ESBL, extended spectrum beta-lactamase.

Table 5. Antibiotic Resistance Rates in First-onset and Recurrent Cases of Urinary Tract Infection

\begin{tabular}{lccc}
\hline & $\begin{array}{c}\text { First-onset } \\
(\mathrm{N}=188)\end{array}$ & $\begin{array}{c}\text { Recurrent } \\
(\mathrm{N}=24)\end{array}$ & $\begin{array}{c}P_{-} \\
\text {value }\end{array}$ \\
\hline Amikacin & $1(0.5 \%)$ & $2(8.3 \%)$ & 0.034 \\
Amoxicillin/Clavulanate & $42(22.3 \%)$ & $11(45.8 \%)$ & 0.012 \\
Ampicillin & $111(59.0 \%)$ & $17(70.8 \%)$ & 0.266 \\
Aztreonam & $20(10.6 \%)$ & $2(8.3 \%)$ & 1.000 \\
Cefepime & $20(10.6 \%)$ & $2(8.3 \%)$ & 1.000 \\
Cefotaxime & $21(11.2 \%)$ & $2(8.3 \%)$ & 1.000 \\
Cefoxitin & $6(3.2 \%)$ & $1(4.2 \%)$ & 0.574 \\
Ceftazidime & $21(11.2 \%)$ & $2(8.3 \%)$ & 1.000 \\
Gentamicin & $21(11.2 \%)$ & $4(16.7 \%)$ & 0.497 \\
Imipenem & $0 \%$ & $0 \%$ & \\
Levofloxacin & $17(12.2 \%)$ & $6(33.3 \%)$ & 0.029 \\
Meropenem & $0 \%$ & $0 \%$ & \\
Piperacillin/tazobactam & $43(22.9 \%)$ & $10(41.7 \%)$ & 0.045 \\
Trimethoprim/Sulfamethoxazole & $38(20.2 \%)$ & $7(29.2 \%)$ & 0.312 \\
\hline
\end{tabular}


( $P=0.012)$, levofloxacin $(P=0.029)$, and TZP $(P=0.045)$

(Table 5).

\section{Antibiotic susceptibility depending on presence} or absence of abnormality in renal ultrasonography

Of the 212 pediatric patients, 177 underwent renal ultrasonography. Of these, 128 had normal findings and 49 had abnormal findings. Among those with abnormal findings, there were 41 cases of hydronephrosis (left, $n=33$; right, $n=$ 1 ; both, $n=7$ ), 4 cases of calyceal dilatation, 1 case of pelvis dilatation, 1 case of renal cyst, 1 case of renal abscess, and 1 case of complete duplication of the left kidney. Comparing rates of antibiotic resistance between patients with normal and abnormal findings on renal ultrasonography, we found no antibiotics associated with significant differences in resistance (Table 6).

\section{Discussion}

UTI is a common childhood infectious disease, affecting approximately $3-5 \%$ of girls and $1 \%$ of boys ${ }^{6}$. Failed early diagnosis and timely initiation of appropriate antibiotic treatment may induce renal parenchymal injury, leading to complications such as renal scarring, hypertension, and chronic kidney disease. Children often exhibit non-specific, systemic symptoms, including fever, diarrhea, vomiting,

Table 6. Comparison of Antibiotic Resistance Rates between Normal and Abnormal Kidney Ultrasound

\begin{tabular}{lccc}
\hline & $\begin{array}{c}\text { Normal } \\
(\mathrm{N}=128)\end{array}$ & $\begin{array}{c}\text { Abnormal } \\
(\mathrm{N}=49)\end{array}$ & $\begin{array}{c}P_{-} \\
\text {value }\end{array}$ \\
\hline Amikacin & $0(0 \%)$ & $1(2.0 \%)$ & 0.277 \\
Amoxicillin/Clavulanate & $27(21.1 \%)$ & $11(22.4 \%)$ & 0.844 \\
Ampicillin & $73(57.0 \%)$ & $28(57.1 \%)$ & 0.989 \\
Aztreonam & $12(9.4 \%)$ & $4(8.2 \%)$ & 1.000 \\
Cefepime & $12(9.4 \%)$ & $4(8.2 \%)$ & 1.000 \\
Cefotaxime & $13(10.2 \%)$ & $4(8.2 \%)$ & 0.783 \\
Cefoxitin & $3(2.3 \%)$ & $1(2.0 \%)$ & 1.000 \\
Ceftazidime & $12(9.4 \%)$ & $4(8.2 \%)$ & 1.000 \\
Gentamicin & $13(10.2 \%)$ & $7(14.3 \%)$ & 0.437 \\
Imipenem & $0 \%$ & $0 \%$ & \\
Levofloxacin & $11(11.5 \%)$ & $5(13.5 \%)$ & 0.770 \\
Meropenem & $0 \%$ & $0 \%$ & \\
Piperacillin/tazobactam & $25(19.5 \%)$ & $11(22.4 \%)$ & 0.666 \\
Trimethoprim/Sulfamethoxazole & $23(18.0 \%)$ & $10(20.4 \%)$ & 0.709 \\
\hline
\end{tabular}

difficulty in breast feeding tantrum, and abdominal distention. These symptoms can result in incorrect diagnosis with simple febrile disease or enteritis. Children who have fever with unknown cause should undergo differential diagnosis for UTI.

Recently, rates of antibiotic resistance have increased, along with increased extended antibiotic use. These factors result in the emergence of multidrug-resistant organisms. The Infectious Diseases Society of America (IDSA) recommended that doctors should continuously inspect and monitor distribution of local UTI strains, as well antibiotic susceptibility changes in these strains ${ }^{7}$.

In children, incidence of UTI varies depending on age and gender. The gender ratio between boys and girls under 1 year of age was 2.8-5.4:1, showing that boys had a higher incidence. The ratio became 1:10 among children 1-2 years of age, showing that the incidence in girls exceeded that in boys $^{6}$. According to another study in South Korea, the ratio between boys and girls under 1 year of age was 2.55-3.4:1, changing to 1:0.6-1.05 in children age 1 year or older ${ }^{8,9)}$. In the present study, ratios between boys and girls by age were 2.21:1 in children younger than 1 month, 3.31:1 in children 1-12 months old, and 1:1.25 in children 13 months or older, showing that UTI incidence was higher in infants and boys aged 1 year or younger, and girls aged 1 year or older.

When we compared all pediatric patients by age, after dividing them into categories including those younger than 1 month (n=29), 1-12 months ( $\mathrm{n}=138$ ), and 13 months or older $(\mathrm{n}=45), E$. coli showed increasing resistance with age to amoxicillin/clavulanate $(P<0.001)$, ampicillin $(P=0.013)$, levofloxacin $(P=0.050)$, TZP $(P<0.001)$, and TMP/SMX $(P=0.002)$. This may be due to more frequent exposure to antibiotics with age; however, these relationships warrant further investigation.

In a 2001 study on antibiotic susceptibility of $E$. coli, Ko et al., ${ }^{10)}$ reported a $7.9 \%$ rate of resistance to $E$. coli for cefotaxime, whereas the present study found this rate to be 5.4 $\%$ in 2008-2012 and 16.8\% in 2013-2016, showing a significant increasing trend $(P=0.008)$. On the other hand, resistance to TZP significantly decreased over these periods from $40.5 \%$ to $7.9 \%(P<0.001)$. These are suspected to be related with frequency of antibiotic used in the local community, which requires further study.

In a 1999 study on community-acquired UTI infections 
in Spain, Daza et al., ${ }^{11)}$ reported a $0.4 \%$ incidence of ESBLproducing E. coli, while Khurana et al., ${ }^{12)}$ reported a $24.7 \%$ incidence of UTIs caused by ESBL-producing E. coli in India in 2002. In the present study, the frequency of ESBLproducing $E$. coli during the total study period was $10.0 \%$, within the range (3.3-10.8\%) of previous reports ${ }^{5,13,14)}$. When we investigated the frequency of ESBL-producing $E$. coli by period in 2008-2012 and in 2013-2016, we observed a significant increasing trend, from $4.6 \%$ to $15.9 \%$.

In addition, the antibiotic susceptibility test of the ESBL (+) strain of 21 cases showed $100 \%$ resistance to cefotaxime. Of these, 19 cases were clinically improved without antibiotic change, and antibiotics were changed in 2 cases due to no clinical improvement. Therefore, the treatment outcome should be considered the basis of actual clinical improvement as well as antibiotic susceptibility results ${ }^{13}$.

Procop et al., ${ }^{15)}$ reported that ESBL-producing Klebsiella pneumonia also had a lower susceptibility to non- $\beta$-lactam antibiotics including gentamicin $(P<0.001)$, amikacin $(P<$ 0.005), TMP/SMX $(P<0.01)$, and nitrofurantoin $(P<0.001)$ than ESBL non-producing Klebsiella pneumonia. The present study found a lower susceptibility to gentamicin $(P=$ $0.004)$, levofloxacin $(P=0.013)$, and TMP/SMX $(P=0.004)$. Therefore, it is necessary to consider antibiotic susceptibility results when choosing antibiotics for treatment.

Compared to the first UTI onset group, the recurrent UTI group had significantly higher resistance to amikacin ( $P=0.034)$, amoxicillin/clavulanate $(P=0.012)$, levofloxacin $(P=0.029)$, and TZP $(P=0.045)$, likely because the recurrence group had higher rates of urogenital anomalies and exposure to antibiotics. In the present study, 27 patients underwent a voiding cystourethrogram (VCUG), in which 18 patients had normal findings, and 9 patients showed findings consistent with vesicoureteral reflux. Of the 9 cases with vesicoureteral reflux, 3 patients were in the initial UTI group, and 6 patients were in the recurrent UTI group, indicating that patients with recurrent UTIs had more vesicoureteral reflux findings. To confirm the relationship, further investigation of urinary tract anomalies, with more samples through VCUG and 99mTc-dimercaptosuccinic Acid (DMSA) Scans are needed.

Since a higher proportion of patients with abnormal findings on renal ultrasonography had recurrent UTIs, due to issues such as structural urinary tract deformities, com- pared to the normal group, we expected this group would also have a higher rate of antibiotic resistance. However, there were 17 patients (13.28\%) with recurrent UTIs among the 128 patients within the normal group, and 6 patients (6.66\%) among the 49 patients in the abnormal group on renal ultrasonography. Thus, the normal group had a higher proportion of individuals with recurrent UTIs. No antibiotics showed significant differences in resistance when we compared the two groups. Therefore, renal ultrasonography alone was not helpful for determining the rate of antibiotic resistance.

The present study investigated antibiotic susceptibility in pediatric patients with UTI caused by E. coli over a 9year sampling period. Urine samples were obtained in 186 cases using the sterile urine collection bag method, 19 cases using a clean catch midstream urine sample, and 7 cases using the catheterization method. The sterile urine collection bag method was the most commonly used urine sample method. However, there were limitations in analyzing the data of antibiotic resistance according to each urine collection methods.

We found increases in the incidence of ESBL-producing E. coli and resistance to cefotaxime. The cause of the increase in the antibiotic resistance rate should be studied in a multifaceted way. These findings warrant further investigation into the frequency of antibiotic use, and its cumulative use, to study changes in antibiotic susceptibility. In addition to total frequency of antibiotic use in the local community, to determine the relationships between these factors and rates of antibiotic resistance, investigation of exposure history and the frequency of antibiotic exposure in individuals are needed.

\section{Conflicts of interest}

No potential conflict of interest relevant to this article was reported.

\section{References}

1. American Academy of Pediatrics Committee on Quality Improvement Subcommittee on Urinary Tract Infection. Practice para- 
meter : the diagnosis, treatment, and evaluation of the initial urinary tract infection in febrile infants and young children. Pediatrics 1999;103:843-52.

2. Sefton AM. The impact of resistance on the management of urinary tract infections. Int J Antimicrob Agents 2000;16:489-91.

3. Lee SY, Lee JH, Kim JH, Hur JK, Kim SM, Ma SH, et al. Susceptibility tests of oral antibiotics including cefixime against Escherichia coli, isolated from pediatric patients with community acquired urinary tract infections. Korean J Pediatr 2006;49:777-83.

4. Kim NH, Lee JA, Kim YK, Choi EH, Ha IS, Lee HJ, et al. Risk factors of urinary tract infections due to extended spectrum beta-lactamase producing Escherichia coli in children. Korean J Pediatr 2004:47:164-9.

5. Kwon YD, Kim MJ, Kim HU, Oh SH, Song JY, Ko JT, et al. Antibiotic sensitivity pattern of pathogens from children with UTI. J Korean Soc Pediatr Nephrol 2006;10:182-91.

6. Elder JS. Urinary tract infections. In : Kliegman RM, Behrman RE, Jensen HB, Stanton BF, editors. Nelson textbook of pediatrics. 18th ed. Philadelphia: WB Saunders Co, 2007:2223-8.

7. Warren JW, Abrutyn E, Hebel JR, Johnson JR, Schaeffer AJ, Stamm WE. Guidelines for antimicrobial treatment of uncomplicated acute bacterial cystitis and acute pyelonephritis in women. Infectious Diseases Society of America (IDSA). Clin Infect Dis 1999; 29:745-58.

8. Jung HJ, Aum JA, Jung SJ, Huh JW. Different characteristic bet- ween Escherichia coli and non-Escherichea coli urinary tract infection. Korean J Pediatr 2007;50:457-61.

9. Lee YK, Lee HC, Chun JM, Yoon SY, Lee WG, Shin SM. Antibiotic sensitivity to the major causative organisms of acute urinary tract infection in children. Korean J Pediatr 2005;48:760-5.

10. Ko YH, Oh JS, Cho DY, Bea JH, Koh SK. Changes of causative organisms and antimicrobial sensitivity of urinary tract infection between 1979 and 2001. Korean J Urol 2003;44:342-50.

11. Daza R, Gutierrez J, Piedrola G. Antibiotic susceptibility of bacterial strains isolated from patients with community acquired urinary tract infections. Int J Antimicrob Agents 2001;18:211-5.

12. Khurana S, Taneja N, Sharma M. Extended spectrum $\beta$-lactamase mediated resistance in urinary tract isolates of family Enterobacteriaceae. Indian J Med Res 2002;116:145-9.

13. Chi HM, Kwahk JH, Lee JH, Park HW. Incidence of Escherichia coli and its susceptibility to antimicrobials in childhood urinary tract infection. J Korean Soc Pediatr Nephrol 2006;10:18-26.

14. Lee JW, Shin JS, Seo JW, Lee MA, Lee SJ. Incidence and risk factors for extended-spectrum beta-lactamase-producing Escherichia coli in community-acquired childhood urinary tract infection. J Korean Soc Pediatr Nephrol 2004;8:214-22.

15. Procop GW, Tuohy MJ, Wilson DA, Williams D, Hadziyannis E, Hall GS. Cross-class resistance to non- $\beta$-lactam antimicrobials in extended-spectrum $\beta$-lactamase-producing Klebsiella pneumoniae. Am J Clin Pathol 2003;120:265-7. 